\title{
Eosinophilic Gastroenteritis: Clinical Manifestation, Natural Course, and Evaluation of Treatment with Corticosteroids and Vedolizumab
}

\author{
Grandinetti, Tanja ; Biedermann, Luc ; Bussmann, Christian ; Straumann, Alex ; Hruz, Petr
}

\begin{abstract}
BACKGROUND Eosinophilic gastroenteritis (EGE) is a rare, chronic inflammatory condition of the gastrointestinal tract. Little is known about its natural history and treatment outcomes. The aims of our analysis were to describe clinical presentation, response to current medical treatments, and to evaluate the response of refractory EGE to anti-integrin therapy. METHODS Patients with confirmed diagnosis of EGE fulfilling the diagnostic criteria: (1) the presence of gastrointestinal symptoms, (2) dense eosinophilic infiltration of the gastrointestinal mucosa, and (3) exclusion of other conditions leading to gastrointestinal eosinophilia were included in this analysis. In patients non-responding to corticosteroids and/or anti-TNF treatment the integrin blocker vedolizumab was used. RESULTS EGE patients $(\mathrm{n}=$ 22 ) were predominantly female $(63 \%)$ with a median age at diagnosis of 41.5 years. The most frequent symptoms were abdominal pain (100\%), diarrhea (59\%), nausea/vomiting (36\%), and bloating (27\%). No pathognomonic endoscopic alterations were found. Eosinophilic infiltration was observed in the majority of patients in more than one segment. Patients were treated with systemic steroids, topical, and enteral release steroids in 21/22 (95\%) patients, proton pump inhibitors in $7 / 22(32 \%)$, TNF inhibitors in $3 / 22(14 \%)$, and vedolizumab in $4 / 22(18 \%)$ patients. In $3 / 4$ of steroid-refractory patients vedolizumab induced a clinical and histological improvement. CONCLUSIONS The combination of highly variable clinical presentation, subtle endoscopic abnormalities, and involvement of several GI segments undermines the difficulty to diagnose EGE and the need for structured biopsy sampling. Corticosteroids were efficient in the majority of patients to induce remission. Response to the integrin blocker vedolizumab suggests further assessment in refractory cases.
\end{abstract}

DOI: https://doi.org/10.1007/s10620-019-05617-3

Posted at the Zurich Open Repository and Archive, University of Zurich

ZORA URL: https://doi.org/10.5167/uzh-181157

Journal Article

Accepted Version

Originally published at:

Grandinetti, Tanja; Biedermann, Luc; Bussmann, Christian; Straumann, Alex; Hruz, Petr (2019). Eosinophilic Gastroenteritis: Clinical Manifestation, Natural Course, and Evaluation of Treatment with Corticosteroids and Vedolizumab. Digestive Diseases and Sciences, 64(8):2231-2241.

DOI: https://doi.org/10.1007/s10620-019-05617-3 
Eosinophilic Gastroenteritis: Clinical Manifestation, Natural Course and Evaluation of Treatment with Corticosteroids and Vedolizumab

Tanja Grandinetti ${ }^{*}$, Luc Biedermann² ${ }^{*}$, Christian Bussmann ${ }^{4}$, Alex Straumann ${ }^{2,3}$, Petr Hruz ${ }^{1}$

${ }^{1}$ Division of Gastroenterology \& Hepatology, University Hospital Basel, Switzerland

${ }^{2}$ Division of Gastroenterology \& Hepatology, University Hospital Zurich, Switzerland

${ }^{3}$ Swiss EoE Clinic, Division of Gastroenterology, University Hospital Zurich, Switzerland

${ }^{4}$ Institute of Pathology Viollier Basel, Switzerland

*Contributed both equally

Short title: Disease course of EGE

Corresponding Author:

Petr Hruz, MD, PhD

Department of Gastroenterology and Hepatology

University Hospital Basel

Petersgraben 4, 4031 Basel, Switzerland

petr.hruz@usb.ch

Phone number: +416132866 09 


\section{Author contributions}

$\mathrm{PH}$ and $\mathrm{AS}$ conceptualized and designed the clinical question, drafted the initial manuscript; TG and LB evaluated the patient data, drafted the initial manuscript; CB performed histological analysis, critically reviewed the manuscript; All authors read and approved the final manuscript as submitted.

Compliance with ethical standards: The authors have no financial relationships relevant to this article to disclose.

Conflict of Interest: The authors have no conflicts of interest to disclose.

\section{Abbreviations}

EoE Eosinophilic Esophagitis

EGE Eosinophilic Gastroenteritis

EGIDS Eosinophilic Gastro-Intestinal Diseases

HPF $\quad$ high power field

$\lg \mathrm{E} \quad$ Immunoglobulin $\mathrm{E}$

PGA patient global assessment

PPI proton pump inhibitor

TNFa tumor necrosis factor alpha 


\section{Abstract}

\section{Background}

Eosinophilic gastroenteritis (EGE) is a rare, chronic-inflammatory condition of the gastrointestinal tract. Little is known about its natural history and treatment outcomes. The aims of our analysis were to describe clinical presentation, response to current medical treatments and to evaluate the response of refractory EGE to anti-integrin therapy.

\section{Methods}

Patients with confirmed diagnosis of EGE fulfilling the diagnostic criteria: 1) presence of gastrointestinal symptoms, 2) dense eosinophilic infiltration of the gastrointestinal mucosa and 3) exclusion of other conditions leading to gastrointestinal eosinophilia were included in this analysis. In patients non-responding to corticosteroids and/or anti-TNF treatment the integrin blocker vedolizumab was used.

\section{Results}

EGE patients $(n=22)$ were predominantly female $(63 \%)$ with a median age at diagnosis of 41.5 years. The most frequent symptoms were abdominal pain (100\%), diarrhea (59\%), nausea/vomiting (36\%) and bloating (27\%). No pathognomonic endoscopic alterations were found. Eosinophilic infiltration was observed in the majority of patients in more than one segment. Patients were treated with systemic steroids, topical and enteral release steroids in $21 / 22(95 \%)$ patients, proton-pump inhibitors in 7/22 (32\%), TNFa inhibitors in 3/22 (14\%) and vedolizumab in 4/22 (18\%) patients. In 3/4 of steroid-refractory patients vedolizumab induced a clinical and histological improvement.

Conclusions

The combination of highly variable clinical presentation, subtle endoscopic abnormalities and involvement of several Gl segments undermines the difficulty to diagnose EGE and the need for structured biopsy sampling. Corticosteroids were efficient in the majority of patients to 
Disease course of EGE

induce remission. Response to the integrin-blocker vedolizumab suggests further assessment in refractory cases.

Key words: Eosinophilic Gastroenteritis; vedolizumab; steroid-refractory 


\section{Introduction}

Eosinophilic gastroenteritis (EGE) was described 80 years ago by the surgeon Kaijser and represents a chronic, immune-mediated inflammatory disorder with an eosinophil-predominant infiltration of the intestinal wall [1-3]. EGE is a rare, heterogeneous and poorly defined clinical condition, which can involve any segment of the gastrointestinal (GI) tract. So far, epidemiologic studies indicate a low prevalence rate of below 5 patients per $100^{\prime} 000$ inhabitants. EGE can affect both children and adults [4,5]. The clinical presentation with unspecific symptoms may mimic other gastrointestinal diseases, in particular irritable bowel syndrome. As a consequence of the diagnostic challenge, EGE is likely underdiagnosed and the prevalence might be markedly higher. The diagnosis is based on the following criteria: presence of gastrointestinal symptoms, eosinophilic infiltration of one or more segments of the gastro-intestinal tract and exclusion of other causes of intestinal eosinophilia, in particular drug reactions, food allergies, celiac disease, parasitic infections, hypereosinophilic syndromes and inflammatory bowel disease [6]. These conditions should be ruled out before the diagnosis of EGE can be established. So far, no consensus has been reached regarding the histological criteria for diagnosing EGE in general and a threshold defining a pathological count of eosinophils in specific, as the mucosa of the Gl tract, with the exception of the esophagus, harbors eosinophils even under physiological conditions [7-9]. EGE remains therefore a diagnostic challenge for the clinician and for the pathologist. So far, two classifications have been used to classify the disease: The first, the Klein-classification is based on the depth of the eosinophilic infiltration on surgical specimens. It describes three different forms: mucosal disease, muscle layer disease and subserosal disease [1]. The second classification is based on the longitudinal pattern of involvement $[4,5]$. The treatment of EGE is another challenge, as recommendations are currently exclusively based on case reports/case series as no controlled trials are available. Our study has the following four purposes: first to provide a comprehensive description of the clinical and endoscopical features of a respectable cohort of 
EGE patients, second to describe the natural course of the disease over time, third to evaluate the response to the current medical treatments and finally to report the experience of treatment with the gut selective $\alpha 4 \beta 7$ integrin-blocker vedolizumab. 


\section{Methods}

Study Design

We performed a retrospective cohort analysis of patients of the Swiss EoE Clinics, a tertiary referral center for eosinophilic gastro-intestinal diseases (EGIDS). Patients of any age with EGE were identified from the Swiss EoE Database (SEED) and clinical presentation, endoscopic and histological features as well as therapeutic decisions were taken from the patient records. Symptoms, endoscopic and histological alterations were documented prospectively. All patients had previously given their informed consent for inclusion into the (SEED). The study was approved by the local ethic committee (EKNZ 2015-388). In addition, a small series of patients with EGE refractory to, or dependent on, corticosteroids were treated with the $\alpha 4 \beta 7$ integrin antibody vedolizumab (Entyvio), on a compassionate use basis, after signing an informed consent.

\section{Patients and Definitions}

The SEED is a nationwide database with voluntary referral, into which patients with Eosinophilic Gastro-Intestinal Disorders (EGIDS) have been prospectively included since 1989. Currently, the database contains data on 1153 EGIDS patients, of which 43 were initially classified as EGE, and 1110 as Eosinophilic Esophagitis (EoE) (Figure 1). In 1991 and 1995 the first two patients with EGE were included. Inclusion of further EGE patients was between 2003 and november 2017. According to the literature, patients were classified as EGE when the following criteria were fulfilled: 1) presence of gastrointestinal symptoms, 2) dense eosinophilic infiltration in biopsies taken from the gastrointestinal tract and/or high eosinophil numbers in ascites, 3) absence of infectious/parasitic or extra-intestinal diseases. Inclusion criteria for this analysis were confirmed diagnosis of EGE, treatment performed at the Swiss EoE Clinic and a complete documentation of the data. Patients with an incomplete dataset 
and patients with isolated eosinophilic infiltration of the esophagus were excluded from this analysis.

Assessment of endoscopic and histological alterations

Esophago-gastro-duodenoscopy (EGD) and/or ileo-colonoscopy were performed in all patients by a board-certified gastroenterologist (AS). Endoscopic alterations were assessed and biopsies obtained for histological evaluation. Histological analysis of biopsies was performed on $4 \mu \mathrm{m}$ paraffin sections of hematoxylin-eosin stained layered cuts $(\geq 10)$. All levels were surveyed and the eosinophils in the most densely infiltrated area were counted in 5 consecutive hpf (microscope Zeiss Axiophot, Plan-Neofluar 40, ocular magnification 10x, area of microscopic field $0.3072 \mathrm{~mm}^{2}$ ).

Patients treated with vedolizumab

Prior to the initiation of compassionate use of vedolizumab extensive counselling of the patient regarding the mechanism of action, therapeutic experiences in IBD as well as safety data was provided. All patients were informed that there is no evidence from clinical trials or case series on the potential role of this therapeutic principle in patients with EGE. In addition, the rationale for this therapeutic approach based on the molecular mechanisms of action of anti-integrin treatment was discussed. All patients provided oral and signed informed consent to treatment with vedolizumab. Dosing was performed according to standard practice used for treatment in patients with inflammatory bowel disease for induction and maintenance with $300 \mathrm{mg}$ infusions at week 0, 2 and 6 followed by maintenance treatment every 8 weeks. At baseline, clinical symptoms were assessed and Patient Global Assessment (PGA) of overall clinical symptoms was determined by patient inquiry and set to 5 per definition at baseline on a scale from 0-10. During the treatment phase, assessment of the PGA was performed at each clinical visit with the following question: How would you personally judge your overall evolution of clinical 
symptoms during treatment on a scale from $0-10$ if the starting point was set to $5 / 10$. Peripheral blood eosinophil count (normal range: $350 \mathrm{Eos} / \mathrm{mm}^{3}$ ) and eosinophilic cationic protein (ECP) (normal range: $<18 \mu \mathrm{g} / \mathrm{l}$ ) were the main biochemical parameters of interest to determine biochemical response. Baseline and follow up endoscopies were performed according to the original disease localization. Severity grade of endoscopic alterations was assessed by the investigator on a visual analogue scale (VAS) from $0-10$ (with 0 indicating no visual abnormalities and 10 most severe signs of active EGE). In cases where there were several segments with active disease, relative weighing with predominant consideration of the most affected segment was done by the main clinical investigator (AS). Quantitative eosinophilic count in eosinophils per high power field (eos/hpf) in the main involved Gl-segment was used as histological outcome. Clinical, biochemical, endoscopic and histological responses were evaluated at the most recent follow-up in cases where there was ongoing treatment with vedolizumab, or at the time of treatment withdrawal. Follow up endoscopy was performed in all patients at the time of the 4th vedolizumab infusion (+/- 10 days), with the exception of patient 2 where endoscopy was performed prematurely at the time of the third infusion because of ongoing severe abdominal pain.

Data collection and statistical analysis

All data were anonymized. The following data were collected: patient characteristics, baseline disease characteristics, peak eosinophil counts, laboratory findings, stool analysis for parasites, treatment characteristics, disease course.

Data extraction from source data was performed using MS Excel. Descriptive statistics included mean and standard deviation (SD) for metric variables and medians and range for ordinal variables and clinical parameters. 


\section{Results}

\section{Characteristics of EGE cohort}

In our database we identified 22 patients, 8 male and 14 female patients with eosinophilic gastroenteritis fulfilling the established diagnostic criteria as well as the inclusion criteria for this analysis (Figure 1). Patients had a median age of 41.5 years at diagnosis and the median interval between first symptoms and diagnosis was 2.5 year (Table 1). In 9/22 (41\%) patients there was a clinical history of allergies. These patients had a history of atopic conditions including asthma, allergic rhinitis, atopic dermatitis and food allergy. Only two patients had a positive family history for eosinophilic gastrointestinal diseases (EGIDS). All patients with EGE presented with abdominal pain. Other frequent symptoms reported by these patients were diarrhea in 59\% and/or nausea/vomiting in 36\% (Table 1). Mostly, there were no relevant findings on physical examination, except in two patients with ascites. Laboratory tests revealed elevated peripheral eosinophilic count in $59 \%$ of patients. Four patients had a discrete anemia, including one patient with a deep ulceration in the duodenum presenting with melena and a hemoglobin drop to $111 \mathrm{~g} / \mathrm{l}$ on one occasion. Serum level of total $\lg E$ was elevated in 8 of 13 analyzed patients, C-reactive protein in 3 of 12, fecal calprotectine in 4 of 6 and in 8 of 9 analyzed patients the eosinophilic cationic protein (ECP) was increased (median: $47.1 \mu \mathrm{g} / \mathrm{l}$, range: $4.8-107 \mu \mathrm{g} / \mathrm{l})$. Stool examinations and cultures were negative for parasites and other common pathogens.

Initial gastroscopy and ileo-colonoscopy was performed in $82 \%(18 / 22)$ and $82 \%(18 / 22)$ patients respectively. The majority of patients had no, or only subtle, endoscopic abnormalities. The most prominent signs were alterations of the esophageal mucosa in 7 out of 9 patients with histologically confirmed affection of the esophagus. In one patient erythematous alterations in the antrum were observed but with no evidence of eosinophilic infiltration on histological evaluation and two patients were found with duodenitis but with no increased eosinophilic infiltrates. In contrast, one patient with a persistent, deep duodenal 
ulceration had eosinophilic infiltration on repeat upper endoscopy. The most prominent finding in the evaluation of the lower gastrointestinal tract were signs of colitis with erythematous mucosal changes in 6/22 (23\%) patients, sigmadiverticulosis in 4/22 (18\%) patients, with erythematous changes in one patient with eosinophilic infiltration. Two patients had mucosal changes in the terminal ileum and ascending colon of small nodular aspect, which proved to be eosinophilic infiltrates on histological evaluation. However, in 11/22 (50\%) patients a normal appearing mucosa was found. The histological evaluation showed that eosinophilic infiltration could occur in all segments of the gastrointestinal tract, but predominantly in the colon (68\%) and the esophagus (41\%) followed by the duodenum (32\%) and terminal ileum (23\%) (Table 2). At diagnosis, counts ranged between 15 and 150 eos/hpf in the most highly involved locations and the observation of eosinophilic microabscesses, intraepithelial eosinophils and eosinophilic degranulation were considered as additional histological features. Involvement of multiple segments of the gastro-intestinal tract and/or organs was observed in 17 patients (77\%). In our cohort 20 patients suffered from mucosal disease and only 2 patients had serosal affection, with both patients initially presenting with eosinophilic ascites. Longitudinal analysis of the clinical course in 20 patients with mucosal type revealed that $30 \%$ had a single flare, $30 \%$ a recurring course with multiple flares and $40 \%$ suffered from a continuous disease course. Interestingly, more than $50 \%$ of patients with affection of the lower gastro-intestinal tract had a single flare course (Figure 2a). In contrast, when the upper gastro-intestinal tract was affected only $25 \%$ of patients had a single disease course whereas the other patients suffered from either a recurring or continuous disease course. All patients with affection of segments in both the lower and upper gastro-intestinal tract suffered from either a recurring or continuous disease course. An increasing number of involved segments was associated with a recurring or continuous disease course (Figure 2b). Two patients with pancreatic manifestation had intestinal involvement of the colon and one patient of two segments of the upper gastrointestinal tract (esophagus and duodenum). One patient with affection of both the 
lower and upper gastrointestinal tract (colon and stomach) had concomitantly diagnosis of an eosinophilic hepatitis and eosinophilic acalculous cholecystitis. These four patients with pancreatic or hepatitic/cholezystitic manifestation had either a recurring or continuous disease course. One patient with a continuous disease course showed primarily affection of the colon and 39 months later an unusual switch to esophageal involvement was observed.

Treatment was based on severity of symptoms, on localization of affected segments in the gastro-intestinal tract and on clinical experience. Five patients were primarily sent to dietary counselling and dietary restrictions were implemented. In these patients an empirical elimination diet was initiated in a step up process starting with wheat elimination or cow milk elimination and progressing up to a dietary restriction typical for a six-food elimination diet (6FED). However, we did not observe clear improvement of symptoms. In 7/22 patients (32\%) a proton pump inhibitor treatment was initiated, $3 / 22(14 \%)$ were treated with antihistamines and 2/22 (9\%) with a mast cell stabilizer with only mild or no clear evidence of clinical improvement (Table 3). All patients initially on PPI or antihistamine treatment were later switched to corticosteroids, except for one patient with a single flare self-limiting disease course. In total, $21 / 22$ (95\%) patients received at least one cycle of corticosteroid treatment. After the start of steroid treatment a tapering scheme was applied according to clinical assessment to reduce the corticosteroid dose to control for clinical symptoms. 8/21 (38\%) of the patients were treated with topical steroids such as fluticasone (induction scheme for 2 weeks $1 \mathrm{mg}$ twice daily and thereafter maintenance therapy of $250 \mu \mathrm{g}$ twice daily) and 10/21 (48\%) of the patients were treated with enteral release steroids such as budesonide (starting dose of $9 \mathrm{mg}$ which was subsequently tapered according to clinical symptoms) . In 13/21 (62\%) patients prednisone $(0.75-1 \mathrm{mg} / \mathrm{kg}$ body weight) was used. Due to persistent clinical symptoms $8 / 21$ (38\%) patients required continuous, long-term prednisone to maintain remission (5-10 $\mathrm{mg} /$ day). In 4 patients refractory or dependent to steroids, biological therapy was initiated. In 
$3 / 21$ (14\%) patients primarily TNFa inhibitors were started. Two of the 3 patients with a TNFa inhibitor therapy were treated with multiple TNFa inhibitors with only mild clinical response.

\section{Outcome of vedolizumab treatment in treatment-refractory EGE}

In 4 patients with treatment-refractory or steroid-dependent course (including three patients not responsive to TNFa treatment), $\alpha 4 \beta 7$ integrin antibody vedolizumab treatement was initiated. Patient and physician reported outcomes for the four patients with EGE treated with vedolizumab are shown in Figure 4.

\section{Patient 1: 24-year-old man with severe eosinophilic duodenitis}

A 24-year old man with eosinophilic gastroenteritis, diagnosed in 2009 with involvement of the esophagus and duodenum, suffered from chronic upper epigastric pain and dysphagia. The patient's symptoms and a large deep duodenal bulb ulceration were refractory to systemic steroids, budesonide and subsequent anti-TNFa (adalimumab) treatment (Figure 3A). Induction and subsequent maintenance treatment with vedolizumab led to a complete resolution of dysphagia as well as eosinophilic infiltration in the duodenum, whereas epigastric pain did not completely disappear. An endoscopic evaluation after continuous treatment over 12 months revealed a superficial duodenal bulb ulceration (Figure 3B). According to this overall favorable course of disease in the absence of systemic steroids, vedolizumab maintenance treatment is continued for cumulative 21 months.

\section{Patient 2: 28-year-old female with serosal small intestinal involvement}

A 28-year old female patient with severe systemic eosinophilia and ascites, diagnosed with EGE after laparoscopic full-thickness biopsy in 2012, predominantly suffered from non-bloody diarrhea, fatigue, weight loss and abdominal cramps with recurrent relapses during several courses of steroid tapering. After unsuccessful treatment with budesonide, thiopurines and two 
anti-TNFa agents (infliximab and adalimumab) vedolizumab was initiated. Due to failure of symptom control anti-integrin treatment was stopped after the induction phase in the $6^{\text {th }}$ week.

\section{Patient 3: 56-year-old female with eosinophilic colitis}

A 56-year old female patient with chronic abdominal pain as the main clinical complaint was diagnosed with eosinophilic colitis with endoscopically unremarkable appearing mucosa with areas of edema and low vascular pattern (Figure $3 \mathrm{C}$ and D). She developed a steroiddependent disease course with increasing diarrhea. Intolerance to azathioprine and 6mercaptopurine, secondary loss of response to infliximab and certolizumab and finally a primary non-response to adalimumab were observed. Treatment with vedolizumab led to a decrease in non-bloody and painless diarrhea. However, due to relapsing symptoms 4-5 weeks after the vedolizumab infusion, the infusion interval was shortened to 4 weeks with cumulative use of vedolizumab for 35 months. Clinical and histological improvement has been observed up to the present.

\section{Patient 4: 25-year-old female with eosinophilic gastroenteritis}

A 25-year old female patient with early onset of IBS-like abdominal symptoms including abdominal pain and irregular bowel movements presented with new onset dysphagia for a gastroenterological evaluation. A thorough endoscopic examination revealed typical features of EoE (Figure 3E) and nodular infiltrates of the terminal ileum (Figure 3F) and the entire colon. Due to refractory symptoms to budesonide a treatment with vedolizumab was initiated. By the end of week 14 improvements in diarrhea and abdominal pain but not dysphagia were observed. Maintenance treatment with vedolizumab is continuing with cumulative use of vedolizumab for 23 months. 


\section{Discussion}

Eosinophilic gastroenteritis (EGE) is a rare but likely underdiagnosed inflammatory disease of the gastrointestinal tract. Due to the unspecific clinical presentation and the lack of clearly defined histologic criteria EGE is a diagnostic challenge for clinicians as well as for pathologists. We herein report on our clinical experience and therapeutic observations in patients with EGE as well as compassionate use of vedolizumab in four patients with treatment refractory disease course.

The most comprehensive information about the natural course was provided in a French cohort analysis of 43 adult patients which identified three different patterns with single flare, a recurring and continuous disease course of EGE [22]. In our cohort patients with extensive disease and patients with affection of both the lower and upper gastrointestinal tract exhibited a recurring or continuous disease course more often. In contrast, patients with only affection of the lower gastrointestinal tract had a more favorable outcome. Of note, we observed a switch of the involved segments during the disease course in one patient.

Our laboratory results revealed peripheral eosinophilia in $52 \%$ of the patients, a finding also observed by other authors [20-22]. Therefore, absence of peripheral eosinophilia is not sufficient to rule out EGE when it is clinically suspected and it is not reliable as an observational parameter to estimate disease activity, as patients may continue to have elevated eosinophil counts regardless of their histological response to therapy. However, high blood eosinophil counts at diagnosis were previously shown to be significantly associated with a high risk of clinical relapse [22]. Eosinophil degranulation and mediator release by activated eosinophils has been shown in tissue samples from patients with eosinophilic gastroenteritis [23-25]. In our cohort we found elevated serum eosinophilic cationic protein (ECP) in a portion of our patients. However, we did not see any difference in the outcome of the course of disease in these patients. This limited observation does not suggest so far that ECP can be of use for diagnosis and monitoring of EGE. 
Endoscopic evaluation was unremarkable in about half of our patients as was similarly observed by other authors [5]. The most frequent endoscopic finding in our patient cohort was colitis and in the esophagus EoE-like alterations, while the most common gastric and duodenal finding described by other authors is mucosal erythema $[5,26]$. Other unspecific signs can include mucosal hyperemia, thickening of folds, friability, areas of roughening, whitish specks, erosions, superficial ulcers, or nodularity [26]. Astonishingly, in many cases we found eosinophilic infiltration in biopsies from areas of normal endoscopic appearance. This apparent inconsistency may arise from the patchy nature of eosinophilic infiltration and from an infiltration restricted to the subepithelial layers, not visible on endoscopic evaluation. In these circumstances if suspicion of EGE is high, a full-thickness surgical biopsy should be performed. Capsule endoscopy could be an alternative option to search for intestinal abnormalities in the small intestine but the benefits of this approach must outweigh the drawbacks, in particular the impossibility of taking biopsies and the risk of impaction in case of strictures $[27,28]$. To summarize, endoscopy might identify inflamed segments of the intestinal tract, but an unremarkable endoscopy does not exclude an eosinophilic inflammation. We therefore strongly recommend performing a structured biopsy sampling in patients with clinical suspicion of EGE, independent of the endoscopic appearance of the mucosa.

So far, there is no established consensus on a diagnostic threshold with regard to eosinophil count for EGE for the various segments of the gastro-intestinal tract. For the stomach and duodenum in asymptomatic adults, a mean eosinophil count of generally less than 10 eosinophils per hpf in biopsies from different areas is considered normal [7, 8, 29]. In clinic practice, based on prior studies on healthy controls, peak eosinophil counts higher than $30 \mathrm{eos} / \mathrm{hpf}$ in the stomach, $50 \mathrm{eos} / \mathrm{hpf}$ in the duodenum, and $30 \mathrm{eos} / \mathrm{hpf}$ in the large bowel (dependent on location) in an affected segment has been suggested when EGE is suspected [30]. In a review, Collins et al suggested less emphasis on eosinophil quantity and focusing 
more on additional pathologic changes. They also proposed using the term "mucosal eosinophilia" to describe increased numbers of mucosal eosinophils without other histologic alterations and reserving the term "EG/colitis" for cases with additional pathologic changes [31].

Analysis of our treatment data revealed that corticosteroids were the mainstay of therapy in most of our EGE patients. Preferentially, prednisone and budesonide were used for gastro-intestinal as well as swallowed fluticasone for patients with esophageal involvement. To maintain remission, approximately a third of our patients required continuous, long-term prednisone. This observation is comparable with our recently published experience in a large cohort of patients with isolated EoE achieving deep remission (i.e. a composite of clinical, endoscopic and histological remission) on continuous treatment with topical steroids [32].

Besides immunosuppressive drugs such as azathioprine and 6-mercaptopurine, TNFa blockers are used for steroid-refractory or steroid-dependent disease course of chronic inflammatory conditions in the gut and also in several case series in patients with EGE [3335]. Since we observed only mild and frequently only transient clinical and biochemical response to treatment with both, azathioprine or TNFa blockers we performed a therapeutic trial with vedolizumab in 4 patients with refractory or steroid-dependent course of EGE. This monoclonal antibody acts against the $\alpha 4 \beta 7$ integrin heterodimer present on activated lymphocytes, blocks the interaction of this integrin with the mucosal addressin cell adhesion molecule (MAdCAM) 1, inhibits leukocyte binding to endothelial surface and prevents therefore extravasation of these cells into affected tissue. Vedolizumab has been shown to efficiently induce and maintain remission in Crohn's disease and ulcerative colitis with moderate to severe disease course during an observation period of up to 152 weeks [36-40]. In addition to a very favorable side effect profile, the main rationale for choosing vedolizumab in this indication was the fact that the $\beta 7$ subunit is also expressed on eosinophils and that MAdCAM1 (expressed on the endothelial cells of the Gl-tract) seems to be upregulated upon 
inflammation [41]. In our case series clinical response, endoscopical as well as histological improvement was observed in two patients with affection of the lower gastrointestinal tract and one patient with eosinophilic duodenitis. Due to failure of symptom control, the anti-integrin treatment was stopped after the induction phase in one patient with serosal type of disease presenting with ascites. Partial response to treatment with vedolizumab with improved histology and reduction of steroids in two patients with a long disease course has been recently observed [42]. Thus, blocking the integrin-MAdCAM-1-interaction might be a novel therapeutic target in refractory cases of EGE. However, any conclusions on the efficacy of vedolizumab in EGE need to be drawn with great caution due to the small patient number reported in our case series and the unblinded, not fully standardized collection of efficacy data.

There are several limitations due to the retrospective nature of our analysis of patients with EGE. Some patients referred for an expert opinion experienced a cross-sectional evaluation and therefore the cohort cannot be considered as a fully representative population of patients with EGE. This includes patients lacking clear signs of chronicity with an uncomplicated disease course where no obvious reasons for eosinophilic infiltration of the gastro-intestinal tract have been found and per definition are considered having EGE. Also in our cohort only two patients suffered from the serosal type of EGE. As only these two patients received a full thickness biopsy, we cannot reliably provide information on the transmural affection in our patient cohort. This indicates that the serosal and muscular sub-type of EGE in our cohort may have been underdiagnosed. In addition, a referral of patients suffering from a complicated and treatment refractory disease course, may have led to a positive selection of difficult to treat patients and therefore the efficacy of some treatments cannot be conclusively assessed and may in fact even have been underestimated.

\section{Conclusion}


Our cohort of this rare and insufficiently understood gastrointestinal disease represents one of the largest observations. EGE is a patchy disease with variable eosinophilic infiltration of all layers of the Gl tract. This suggests that diagnosis of EGE should be suspected in patients with long lasting unexplained chronic gastrointestinal symptoms and a standardized thorough endoscopic as well as histological evaluation of normal appearing intestinal segments should be performed Treatment with corticosteroids is central in the treatment of patients with EGE. Although vedolizumab induced clinical response in some patients with treatment refractory disease course of EGE, the efficacy of this drug needs to be evaluated for this indication with a standardized protocol in a prospective multicenter study. 


\section{References}

1. Klein NC, Hargrove RL, Sleisenger MH, et al. Eosinophilic gastroenteritis. Medicine (Baltimore). 1970;49:299-319.

2. R K. Zur Kenntnis der allergischen Affektionen des Verdaungskanal von Standpunkt des Chirurgen aus. Arch Klin Chir 1937;188.

3. Yan BM, Shaffer EA. Primary eosinophilic disorders of the gastrointestinal tract. Gut. 2009;58:721-32.

4. Mansoor E, Saleh MA, Cooper GS. Prevalence of Eosinophilic Gastroenteritis and Colitis in a Population-Based Study, From 2012 to 2017. Clin Gastroenterol Hepatol. 2017; 15:1733-41.

5. Reed C, Woosley JT, Dellon ES. Clinical characteristics, treatment outcomes, and resource utilization in children and adults with eosinophilic gastroenteritis. Dig Liver Dis. 2015;47:197-201.

6. Walker MM, Potter M, Talley NJ. Eosinophilic gastroenteritis and other eosinophilic gut diseases distal to the oesophagus. Lancet Gastroenterol Hepatol. 2018;3:271-80.

7. DeBrosse CW, Case JW, Putnam PE, et al. Quantity and distribution of eosinophils in the gastrointestinal tract of children. Pediatr Dev Pathol. 2006;9:210-8.

8. Lwin T, Melton SD, Genta RM. Eosinophilic gastritis: histopathological characterization and quantification of the normal gastric eosinophil content. Mod Pathol. 2011;24:55663.

9. Matsushita $\mathrm{T}$, Maruyama R, Ishikawa $\mathrm{N}$, et al. The number and distribution of eosinophils in the adult human gastrointestinal tract: a study and comparison of racial and environmental factors. Am J Surg Pathol. 2015;39:521-7. 
10. Chen MJ, Chu CH, Lin SC, et al. Eosinophilic gastroenteritis: clinical experience with 15 patients. World J Gastroenterol. 2003;9:2813-6.

11. Talley NJ, Shorter RG, Phillips SF, et al. Eosinophilic gastroenteritis: a clinicopathological study of patients with disease of the mucosa, muscle layer, and subserosal tissues. Gut. 1990;31:54-8.

12. Chang JY, Choung RS, Lee RM, et al. A shift in the clinical spectrum of eosinophilic gastroenteritis toward the mucosal disease type. Clin Gastroenterol Hepatol. 2010;8:669-75; quiz e88.

13. Milic S, Poropat G, Malic D, et al. A case of postpartum eosinophilic gastroenteritis and review of the literature. Dig Dis. 2012;30:232-5.

14. Singh PS, Kushwaha SR. Eosinophilic ascitis, an unusual presentation of eosinophilic gastro-enteritis. J Indian Med Assoc. 2014;112:117-8, 23.

15. Baek MS, Mok YM, Han WC, et al. A patient with eosinophilic gastroenteritis presenting with acute pancreatitis and ascites. Gut Liver. 2014;8:224-7.

16. Han SG, Chen Y, Qian ZH, et al. Eosinophilic gastroenteritis associated with eosinophilic cystitis: Computed tomography and magnetic resonance imaging findings. World J Gastroenterol. 2015;21:3139-45.

17. Issa $\mathrm{H}$, Bseiso $\mathrm{B}, \mathrm{Al}-$ Salem $\mathrm{AH}$. Eosinophilic enteritis presenting as a perforated duodenal ulcer. Endoscopy. 2011;43 Suppl 2 UCTN:E358-9.

18. Siaw EK, Sayed K, Jackson RJ. Eosinophilic gastroenteritis presenting as acute gastric perforation. J Pediatr Gastroenterol Nutr. 2006;43:691-4.

19. Zhou HC, Lai C, Yang L. Eosinophilic gastroenteritis with involvement of the urinary bladder. Pediatr Radiol. 2014;44:1454-7.

20. Kinoshita Y, Furuta K, Ishimaura N, et al. Clinical characteristics of Japanese patients with eosinophilic esophagitis and eosinophilic gastroenteritis. J Gastroenterol. 2013;48:333-9 
21. Ko HM, Morotti RA, Yershov $O$, et al. Eosinophilic gastritis in children: clinicopathological correlation, disease course, and response to therapy. Am J Gastroenterol. 2014;109:1277-85.

22. Pineton de Chambrun G, Gonzalez F, Canva JY, et al. Natural history of eosinophilic gastroenteritis. Clin Gastroenterol Hepatol. 2011;9:950-6 e1.

23. Bischoff SC, Mayer J, Nguyen QT, et al. Immunnohistological assessment of intestinal eosinophil activation in patients with eosinophilic gastroenteritis and inflammatory bowel disease. Am J Gastroenterol. 1999;94:3521-9.

24. Kephart GM, Alexander JA, Arora AS, et al. Marked deposition of eosinophil-derived neurotoxin in adult patients with eosinophilic esophagitis. Am J Gastroenterol. 2010;105:298-307.

25. Torpier G, Colombel JF, Mathieu-Chandelier C, et al. Eosinophilic gastroenteritis: ultrastructural evidence for a selective release of eosinophil major basic protein. Clin Exp Immunol. 1988;74:404-8.

26. Zhang L, Duan L, Ding S, et al. Eosinophilic gastroenteritis: clinical manifestations and morphological characteristics, a retrospective study of 42 patients. Scand $J$ Gastroenterol. 2011;46:1074-80.

27. Attar A, Cazals-Hatem D, Ponsot P. Videocapsule endoscopy identifies stenoses missed by other imaging techniques in a patient with eosinophilic gastroenteritis. Clin Gastroenterol Hepatol. 2011;9:A28.

28. Pasha SF, Leighton JA, Williams JW, et al. Capsule retention in a patient with eosinophilic gastroenteritis mimicking diaphragm disease of the small bowel. Endoscopy. 2009;41 Suppl 2:E290-1.

29. Talley NJ, Walker MM, Aro P, et al. Non-ulcer dyspepsia and duodenal eosinophilia: an adult endoscopic population-based case-control study. Clin Gastroenterol Hepatol. 2007;5:1175-83. 
30. Egan M, Furuta GT. Eosinophilic gastrointestinal diseases beyond eosinophilic esophagitis. Ann Allergy Asthma Immunol. 2018;121:162-7.

31. Collins MH, Capocelli K, Yang GY. Eosinophilic Gastrointestinal Disorders Pathology. Front Med (Lausanne). 2017;4:261.

32. Greuter T, Bussmann C, Safroneeva E, et al. Long-Term Treatment of Eosinophilic Esophagitis With Swallowed Topical Corticosteroids: Development and Evaluation of a Therapeutic Concept. Am J Gastroenterol. 2017;112:1527-35.

33. Muir A, Surrey L, Kriegermeier A, et al. Severe Eosinophilic Gastroenteritis in a Crohn's Disease Patient Treated With Infliximab and Adalimumab. Am J Gastroenterol. $2016 ; 111: 437-8$

34. Redondo-Cerezo E, Cabello MJ, Gonzalez Y, et al. Eosinophilic gastroenteritis: our recent experience: one-year experience of atypical onset of an uncommon disease. Scand J Gastroenterol. 2001;36:1358-60.

35. Turner D, Wolters VM, Russell RK, et al. Anti-TNF, infliximab, and adalimumab can be effective in eosinophilic bowel disease. J Pediatr Gastroenterol Nutr. 2013;56:492-7.

36. Amiot A, Grimaud JC, Peyrin-Biroulet L, et al. Effectiveness and Safety of Vedolizumab Induction Therapy for Patients With Inflammatory Bowel Disease. Clin Gastroenterol Hepatol. 2016;14:1593-601 e2.

37. Feagan BG, Rutgeerts $\mathrm{P}$, Sands $\mathrm{BE}$, et al. Vedolizumab as induction and maintenance therapy for ulcerative colitis. N Engl J Med. 2013;369:699-710.

38. Loftus EV, Jr., Colombel JF, Feagan BG, et al. Long-term Efficacy of Vedolizumab for Ulcerative Colitis. J Crohns Colitis. 2017;11:400-11.

39. Sandborn WJ, Feagan BG, Rutgeerts $P$, et al. Vedolizumab as induction and maintenance therapy for Crohn's disease. N Engl J Med. 2013;369:711-21.

40. Vermeire S, Loftus EV, Jr., Colombel JF, et al. Long-term Efficacy of Vedolizumab for Crohn's Disease. J Crohns Colitis. 2017;11:412-24. 
41. Rieder F, Biancani P, Harnett K, et al. Inflammatory mediators in gastroesophageal reflux disease: impact on esophageal motility, fibrosis, and carcinogenesis. Am J Physiol Gastrointest Liver Physiol. 2010;298:G571-81.

42. Kim HP, Reed CC, Herfarth HH, et al. Vedolizumab Treatment May Reduce Steroid Burden and Improve Histology in Patients With Eosinophilic Gastroenteritis. Clin Gastroenterol Hepatol. 2018;16:1992-4. 
Table 1: Demographic and disease specific characteristics of patients with EGE $(n=22)$

\begin{tabular}{|c|c|c|}
\hline Variable & $\mathbf{n}$ & $\%$ \\
\hline Gender: male/female & $8 / 14$ & $37 / 63$ \\
\hline Median age at onset of symptoms (range) & \multicolumn{2}{|c|}{$31(6-74)$} \\
\hline Median age at diagnosis (range) & \multicolumn{2}{|c|}{$41.5(13-78)$} \\
\hline $\begin{array}{l}\text { Median diagnostic delay (time between onset of symptoms and diagnosis) } \\
\text { (range) }\end{array}$ & \multicolumn{2}{|c|}{$\begin{array}{l}2.5(0-40 \\
\text { years })\end{array}$} \\
\hline Median duration of disease in years (range) & \multicolumn{2}{|c|}{$\begin{array}{c}8.5(1-26 \\
\text { years })\end{array}$} \\
\hline Positive family history for EGIDS* & 2 & 9 \\
\hline Clinical history of allergies & 9 & 41 \\
\hline Atopic Diathesis & 6 & 38 \\
\hline Allergic Rhinoconjunctivitis & 3 & 14 \\
\hline Asthma & 6 & 28 \\
\hline Atopic dermatitis & 1 & 5 \\
\hline Urticaria & 1 & 5 \\
\hline Oral allergy syndrome & 1 & 5 \\
\hline Celiac Disease & 1 & 5 \\
\hline \multicolumn{3}{|l|}{ Clinical and laboratory characteristics } \\
\hline Symptoms (may be more than one category per patient) & $\mathbf{n}$ & $(\%)$ \\
\hline Abdominal pain & 22 & 100 \\
\hline Upper abdominal pain & 10 & 45 \\
\hline Lower and mid abdominal pain & 12 & 55 \\
\hline Diarrhea & 13 & 59 \\
\hline Nausea/Vomiting & 8 & 36 \\
\hline Reflux & 3 & 14 \\
\hline Dysphagia & 3 & 14 \\
\hline Bloating & 6 & 27 \\
\hline Loss of appetite/Weight loss & 4 & 18 \\
\hline
\end{tabular}




\begin{tabular}{|l|c|c|}
\hline Fatigue & 4 & $\mathbf{1 8}$ \\
\hline Fever & 1 & $\mathbf{5}$ \\
\hline Gastrointestinal bleeding & 1 & $\mathbf{5}$ \\
\hline Constipation & 1 & $\mathbf{5}$ \\
\hline Laboratory parameters & 13 & $\mathbf{5 9}$ \\
\hline Blood eosinophilia $\left(\right.$ normal range: $<350$ Eos $\left./ \mathrm{mm}^{3}\right)$ & 8 & $\mathbf{6 2}$ \\
\hline Elevated total serum immunoglobulin E $(\text { normal range: }<100 \mathrm{IU} / \mathrm{ml})^{\mathrm{a}}$ & 3 & $\mathbf{2 5}$ \\
\hline Elevated C- reactive protein (normal range: $<10 \mathrm{mg} / \mathrm{ml})^{\mathrm{b}}$ & 4 & $\mathbf{6 6}$ \\
\hline Elevated Fecal calprotectin (normal range: $<50 \mu \mathrm{gg} / \mathrm{g} \mathrm{stool})^{\mathrm{c}}$ & 8 & $\mathbf{8 9}$ \\
\hline Elevated Eosinophil Cationic Protein $\left(\mathrm{normal} \mathrm{range}:<18 \mu \mathrm{gg} / \mathrm{l}^{\mathrm{d}}\right.$ & 4 & $\mathbf{1 8}$ \\
\hline Anemia & &
\end{tabular}

* EGIDS = Eosinophilic Gastrointestinal Diseases

${ }^{a}$ analysis was available for 13 patients; ${ }^{b}$ for 12 patients, ${ }^{c}$ for 6 patients and ${ }^{d}$ for 9 patients 
Table 2: Involved intestinal segments and organs at baseline examination

\begin{tabular}{|l|c|c|}
\hline $\begin{array}{l}\text { Organ tract involved at baseline examination (may be more than one category per } \\
\text { patient) }\end{array}$ & $\mathbf{n}$ & (\%) \\
\hline Esophagus & 9 & $\mathbf{4 1}$ \\
\hline Stomach & 3 & $\mathbf{1 4}$ \\
\hline Duodenum & 7 & $\mathbf{3 2}$ \\
\hline Terminal ileum & 5 & $\mathbf{2 3}$ \\
\hline Large intestine (Colon) & 15 & $\mathbf{6 8}$ \\
\hline Peritoneum & 2 & $\mathbf{9}$ \\
\hline Pancreas & 3 & $\mathbf{1 4}$ \\
\hline Eosinophilic Hepatitis and eosinophilic acalculous Cholezystitis & 1 & $\mathbf{5}$ \\
\hline More than one gastrointestinal segment and/or organ involved & 17 & $\mathbf{7 7}$ \\
\hline
\end{tabular}


Table 3: Drug treatment of patients with Eosinophilic Gastroenteritis

\begin{tabular}{|c|c|c|}
\hline Medication & $\mathbf{n}$ & (\%) \\
\hline Corticosteroids & 21 & 95 \\
\hline Topical esophageal steroids (swallowed fluticasone or budesonide) & 8 & 36 \\
\hline Enteral release steroids (budesonide) & 10 & 45 \\
\hline Systemic steroids & 13 & 59 \\
\hline Proton Pump Inhibitor & 9 & 41 \\
\hline Antihistamines & 3 & 14 \\
\hline Mast cell stabilizer & 2 & 9 \\
\hline Azathioprine/6-Mercaptopurine (6-MP) ${ }^{a}$ & 5 & 23 \\
\hline Methotrexate & 1 & 5 \\
\hline Antibiotics (ciprofloxacine, metronidazole) ${ }^{\mathrm{b}}$ & 7 & 32 \\
\hline TNFa Blocker (Infliximab, Adalimumab, Certolizumab) ${ }^{c}$ & 3 & 14 \\
\hline Integrin Antagonist (Vedolizumab) & 4 & 18 \\
\hline
\end{tabular}

${ }^{a}$ two patients with side effects of hepatopathy on treatment with azathioprine

${ }^{\mathrm{b}}$ all patients received combination of ciprofloxacine and metronidazole

${ }^{c}$ one patiens received Ada, one patient received Ifx and Ada and one patient received Ada, Ifx and Cer 


\section{Figure Legends}

\section{Figure 1:}

Flowchart of screened patients currently enrolled in the SEED.

\section{Figure 2:}

Disease course in patients affected with the mucosal type of EGE $(n=20)$. Correlation between disease course of EGE and (A) anatomical distribution upper (up) vs lower (lo) vs upper and lower (up/lo) gastrointestinal tract and (B) number of involved gastrointestinal segments.

\section{Figure 3:}

(A) Endoscopic picture the duodenal bulb of a 24 year old male patient with eosinophilic duodenitis showing a deep ulceration, inflammation and severe stricture. Follow up endoscopy (B) after one year on continuous treatment with vedolizumab showing discrete inflammation, superficial erosions and moderate stricture. (C) Endoscopic picture of the colon of a 56 year old female patient with eosinophilic colitis showing a mainly unremarkable appearing mucosa with areas of edema and low vascular pattern with a (D) representative histologic picture of the colon mucosa with eosinophilic colitis showing a dense infiltration with eosinophils. (HE staining; original magnification 320x). (E) Endoscopic picture of a 27 year old female patient with eosinophilic gastroenteritis with involvement of the esophagus showing edema, white exudates, furrows and subtle trachealization and $(F)$ of the terminal ileum showing an intact mucosa with small, prominent nodules. 


\section{Figure 4:}

Patient- and physician-reported outcomes at baseline and at follow up of 4 patients treated with the integrin blocker vedolizumab.

PGA = patient global assessment; endoscopic activity in the esophagus based on EREFscoring; histologic activity with peak eosinophil count; peripheral blood eosinophilia (eosinophils/mm3); serum eosinophil cationic protein (ECP) 